\title{
Diagnostic Value of New Features for Endometritis under Hysteroscopy
}

\author{
Yue Zhang', Yi Yang ${ }^{2, *}$ \\ ${ }^{1}$ Sixth Medical Center of PLA General Hospital, Beijing, China. \\ ${ }^{2}$ Institute of Defence Economics and Management, Central University of Finance and Economics, Beijing, China.
}

\begin{abstract}
How to cite this paper: Yue Zhang, Yi Yang. (2022) Diagnostic Value of New Features for Endometritis under Hysteroscopy. International Journal of Clinical and Experimental Medicine Research, 6(1), 10-15.

DOI: 10.26855/ijcemr.2022.01.003
\end{abstract}

Received: September 28, 2021

Accepted: October 23, 2021

Published: November 22, 2021

*Corresponding author: Yi Yang, Institute of Defence Economics and Management, Central University of Finance and Economics, Beijing, China. Email: yiyang86@msn.com

\begin{abstract}
Objective: To evaluate the value of neovascularization, endometrial irregular hyperplasia and endometrial polypoid hyperplasia in diagnosis of chronic endometritis. Design: retrospective cohort study. Setting: University teaching hospital. Patient(s): A consecutive series of 222 cases of diagnostic hysteroscopy. Intervention(s): Endometrial biopsy specimens were obtained after hysteroscopy for routine histology and immunohistochemistry for plasma cells using a CD138 epitope. Main Outcome Measure(s): Neovascularization, endometrial polypoid hyperplasia, endometrial irregular hyperplasia of the hysteroscopic features in the diagnosis of CE. Result(s): A total of 670 infertile patients underwent hysteroscopy, and the incidence of microscopic neovascularization was about $22.4 \%$. Among them, 222 patients underwent pathological examination and CD138 immunoassay. 94 patients were diagnosed Chronic endometritis pathologically. Neovascularization was moderately consistent with CE. (Kappa coefficient was 0.46, 95\% 0.342-0.578, P < 0.05; $\mathrm{OR}=8.153$ ), there was no strong correlation between endometritis, polypoid hyperplasia and irregular hyperplasia. The sensitivity, specificity, positive predictive value and negative predictive value of neovascularization were $71.27 \%, 75 \%, 67.68 \%$ and $78.05 \%$, respectively, and the diagnostic accuracy was $73.42 \%$. Conclusion: There was a moderate consistency between neovascularization and CE, which could be used as a characteristic index for physicians to judge chronic endometritis and improve the accuracy of CE diagnosis by hysteroscopy. Irregular hyperplasia and polypoid hyperplasia of uterus have no statistical significance for the diagnosis of chronic endometrium, but whether they affect embryo implantation rate should be discussed in the future.
\end{abstract}

\section{Keywords}

Neovascularization, Endometrial Irregular Hyperplasia, Endometrial polypoid hyperplasia, Chronic Endometritis

Chronic endometritis (CE) is currently considered to be a pathological condition in which the endometrial stroma is infiltrated by inflammatory cells [1]. In previous clinical practice, CE was often ignored due to slight lack of specificity of symptoms, difficult diagnosis and time-consuming diagnosis. In recent years, studies have reported that the incidence of CE in infertility patients is gradually increasing, and CE has become one of the common diseases in the reproductive field [2]. According to relevant studies, the incidence of CE in infertility patients is $0.2 \%-46 \%$; In 2010, Makrakis et al. found that CE incidence was as high as $42 \%$ in women with repeated transplant failures; 
Bouet et al. found that the incidence of CE in patients with recurrent abortion in early pregnancy was 27\% [3-5].

At present, the diagnosis of CE mainly relies on hysteroscopy, pathological examination, and CDl38 immunohistochemical staining, in which the presence of plasma cell infiltration in the endometrial stroma by histological staining is the gold standard for the diagnosis of CE. Hysteroscopy is recommended by scholars for its intuitive and comprehensive observation and evaluation of endometrium. At present, the characteristics of CE under hysteroscopy are microscopic polyps, interstitial edema, and patchy hyperemia [6-9]. However, Dongmei Song, M. Ed [10] found that the accuracy of hysteroscopy in diagnosing CE was only 67\%, which could not replace histological examination as a diagnostic tool. The reason is that the endometrium presents diversity under hysteroscopy, such as neovascularization, endometrial polypoid hyperplasia, and endometrial irregular hyperplasia. However, the diagnostic value of these clinical phenotypes for CE is not clear. This paper focuses on the diagnostic value of these characteristics in chronic endometritis under hysteroscopy.

\section{Data and Methods}

\subsection{Data}

Subjects: Infertility patients who underwent hysteroscopy in the Reproductive Center of the Sixth Medical Center of the PLA General Hospital from August 2018 to April 2021 were collected. The age range was 28-47 years, $36.1 \pm 5.55$ years. Inclusion criteria: patient age $<50$ years; having a normal sexual life; the couple had no major diseases and active infectious diseases, and their chromosomes were normal. Exclusion criteria: (1) The patient has endocrine diseases and other diseases resulting in infertility; (2) Having history of intrauterine operation in the past two months, b-mode ultrasonography showed no uterine malformation, fibroids, mediastinum, etc. Tubal effusion; (3) Having taken birth control pills or other hormonal drugs in the last 3 months; (4) intrauterine adhesion; (5) the male has serious semen abnormalities resulting in infertility. The study was conducted with the informed consent of the participating patients.

\subsection{Methods}

Hysteroscopy was performed during endometrial proliferation. Xenon cold light source, TV camera and monitoring system from Germany STORZ company are used. Hysteroscopy adopts the hard hysteroscopy of STORZ Company TROPHY and the soft hysteroscopy of OLYMPUS Company HYF-XP fiber. Normal saline was used as the distention medium. The initial distention pressure was set at $60-80 \mathrm{~mm} \mathrm{Hg}$ under anesthesia, and the pressure was adjusted during operation. All surgical procedures were performed by the same group of physicians, with clear field of vision and close observation of intimal changes. During hysteroscopy, each patient was kept and recorded with video for pathological patients.

\subsection{Main outcome measures}

Neovascularization (see Figure 1 and Figure 2): Dilated or ruptured superficial and subepithelial blood vessels on the endometrium.

Endometrial irregular hyperplasia (see Figure 3): This is a diffuse polypoid appearance in the uterine cavity, characterized by soft protrusions, uneven thickness, and no apparent polyp.

Endometrial polypoid hyperplasia (see Figure 4): elongated conical or fingerlike, with prominent pedicles, glands, and stroma but no evident vascular axis.

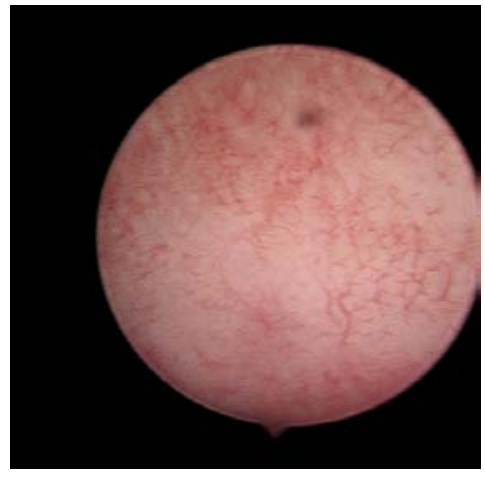

Figure 1. Small scattered neovascularization.

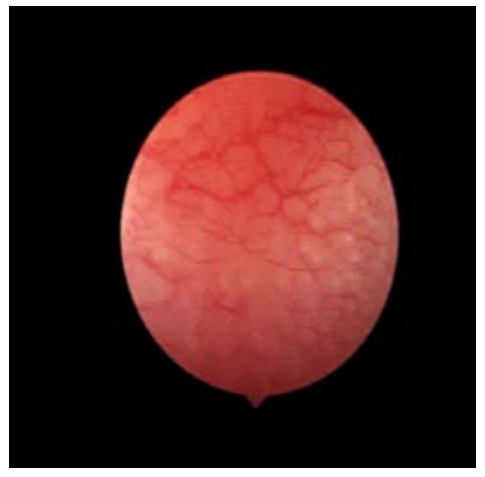

Figure 2. Large hyperplasia of blood vessels. 


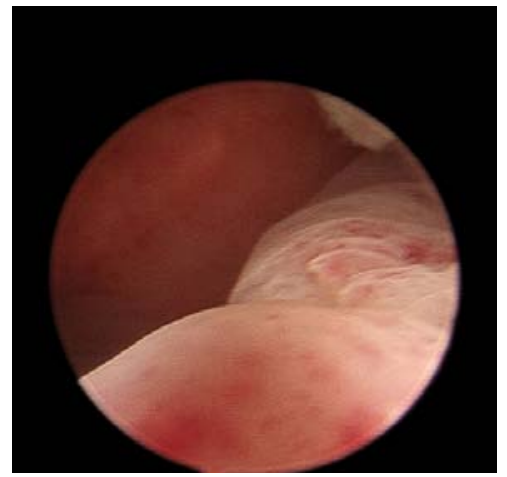

Figure 3. Endometrial Irregular hyperplasia.

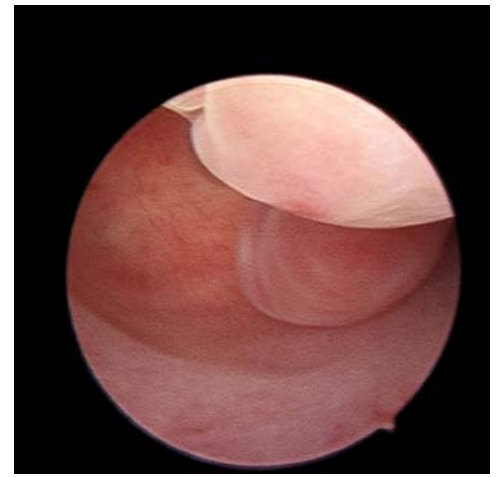

Figure 4. Endometrial Polypoid hyperplasia.

Chronic endometritis: Plasma cells can be seen under the tissue examination microscope. Chronic endometritis is diagnosed by positive HE staining or CD138 immunohistochemistry.

\subsection{Statistical methods}

SPSS 22.0 software is adopted for statistical analysis. The measurement data of normal distribution were expressed as mean \pm standard deviation $(X \pm S)$, and independent sample $\mathrm{T}$ test was used for analysis between groups. The consistency between hysteroscopic neovascularization and pathological diagnosis (Kappa value) was compared. When $0.4<$ Kappa $\leq 0.7$, the consistency between the two diagnostic methods was considered to be moderate. HE staining and/or CD138 immunohistochemical diagnosis were used as reference.

The Peason $\mathrm{X}^{2}$ independence test was used for correlation analysis of categorical variables, and multivariate non-conditional Logistic regression analysis was performed for all variables with $\mathrm{P}<0.05$ in univariate analysis. $\mathrm{P}<0.05$ was considered as statistically significant difference.

\section{Results}

A total of 670 infertile patients underwent hysteroscopy, and the incidence of microscopic neovascularization was about $22.4 \%$. In 222 patients, the uterine cavity was scratched with a small scraper and pathological examination was performed. Histopathological examination and immune CD138 detection were performed. Chronic endometritis was diagnosed pathologically in 94 patients (see Table 1). The Kappa coefficient of consistency between CE diagnosis of neovascularization and pathology was 0.46 (95\%CI interval $0.342-0.578, \mathrm{P}<0.05)$, indicating moderate consistency between the two (see Table 2). The sensitivity, specificity, positive predictive value and negative predictive value of $\mathrm{CE}$ in neovascularization diagnosis were $71.27 \%, 75 \%, 67.68 \%$ and $78.05 \%$, respectively, and the diagnostic accuracy was $73.42 \%$. Endometrial polypoid hyperplasia, irregular hyperplasia and endometritis are not strongly correlated.

Among 222 patients, 94 cases were pathologically diagnosed as CE, 67 cases of neovascularization $(71.27 \%$, $\mathrm{OR}=8.153$, 95\%CI4.377-15.186, $\mathrm{P}<0.05), 24$ cases of polypoid hyperplasia $(25.53 \%$, OR=1.772, 95\%CI0.840-3.739 $\mathrm{P}=0.133)$ and uneven thickening in 25 cases $(26.60 \%$, OR=0.744 95\%CI0.973-1.096 P $=0.291)$.

Table 1. Consistency analysis of neovascularization, irregular hyperplasia, polypoid hyperplasia and CE

\begin{tabular}{|c|c|c|c|c|}
\hline & \multicolumn{2}{|c|}{ The pathologic result } & \multirow[t]{2}{*}{ Kappa value } & \multirow[t]{2}{*}{$\mathrm{P}$} \\
\hline & $(+)$ & $(-)$ & & \\
\hline neovascularization & & & 0.46 & $<0.001$ \\
\hline$(+)$ & 67 & 32 & & \\
\hline$(-)$ & 27 & 96 & & \\
\hline irregular hyperplasia & & & -0.024 & 0.705 \\
\hline$(+)$ & 25 & 37 & & \\
\hline$(-)$ & 69 & 91 & & \\
\hline polypoid hyperplasia & & & 0.056 & 0.358 \\
\hline$(+)$ & 24 & 26 & & \\
\hline$(-)$ & 70 & 102 & & \\
\hline
\end{tabular}


Table 2. The possibility of CE in neovascularization, endometrial irregular hyperplasia and endometrial polypoid hyperplasia

\begin{tabular}{ccccc}
\hline & $\mathrm{B}$ & $\mathrm{p}$-value & OR & $95 \%$ CI \\
\hline neovascularization & 2.098 & 0.000 & 8.153 & $4.377-15.186$ \\
polypoid hyperplasia & 0.572 & 0.133 & 1.772 & $0.840-3.739$ \\
irregular hyperplasia & -0.296 & 0.404 & 0.744 & $0.371-1.490$ \\
Age, yr & 0.032 & 0.291 & 1.033 & $0.973-1.096$ \\
constant & -2.521 & 0.030 & .080 & \\
\hline
\end{tabular}

\section{Discussion}

Normal endometrium carries physiological events such as menstruation, ovulation, implantation and pregnancy, and periodic injury and healing occur every month, all of which are characteristics of self-limiting inflammation [11-12]. Leukocytes, neutrophils, eosinophils, macrophages, NK cells and other inflammatory cells normally exist in the endometrium, and their proportion gradually increases from the proliferative stage to the secretory stage, helping implantation and pregnancy. However, there are no plasma cells in normal intima. Therefore, the presence of plasma cells is the gold standard for diagnosing CE. The diagnostic accuracy of CE under hysteroscopy at proliferation stage was better than that at secretion stage. At present, the main cause of CE disease is bacterial or other microbial infection. Antibiotics are the main treatment method, and the effective rate is about $80 \%-90 \%$. At present, the universally accepted criteria for the diagnosis of endometritis under hysteroscopy are: endometrial congestion: (1) Strawberry sign: large endometrial congestion, with large and white punctured openings in the middle gland. (2) Focal congestion: small area of endometrial congestion. (3) Hemorrhagic spot: dark/brownish red hemorrhagic spot with sharp and irregular boundary, which may be connected with capillaries. (4) Microscopic polyps: diameter less than 1mm; Vascular axles of connective tissue are attached or run along the entire endometrial surface. (5) Interstitial edema: the endometrium is thick and edema at the follicular stage.

Dongmei Song, M. Ed [10]. A study of 1189 patients undergoing hysteroscopy and pathological diagnosis of CE in 2019 found that CD138 (+) was detected in 322 cases (27.1\%) of 1189 patients. The characteristics of hysteroscopy were as follows: endometrial congestion: 169 of 322 cases (52.5\%); Endometrial interstitial edema :27 cases 322 cases (8.4\%); Micropolyps: 11 of 322 (3.4\%). The sensitivity, specificity, positive and negative predictive value and diagnostic accuracy of hysteroscopy were $59.3 \%, 69.7 \%, 42.1 \%, 82.8 \%$ and $66.9 \%$, respectively. Conclusions: The detection of endometrial congestion, microscopic polyps or endometrial interstitial edema during hysteroscopy should be cautious of CE, but the overall accuracy of hysteroscopy in diagnosing CE is only 67\%, so it should not replace the choice of histological examination as a diagnostic tool.

Normal intimal vessels are known as spiral arterioles perpendicular to the intimal epithelium. Hysteroscopic large neovascularization is parallel to the epithelium. Previous studies have shown that the quantity and quality of leukocyte recruitment, related cytokines and blood vessels varies over time throughout the menstrual cycle and pregnancy [11-21]. Vascular changes are mainly composed of vasoconstriction, vasodilation, increased capillary permeability and angiogenesis. Interleukin 1, interleukin 6, interleukin 8 and peptide growth factor play key roles in reproductive physiology and pathology, mainly through prostaglandin synthesis pathway. Bacterial infection can cause changes in cytokines, leading to vascular dilation and abnormal proliferation. Due to the angiogenesis of adenomyosis, neovascularization is characteristic of adenomyosis under endoscopy. Akie Takebayashi [22] found that endometritis in adenomyosis patients was three times as high as that in non-adenomyosis patients, and neovascularization disappeared in adenomyosis patients under hysteroscopy after 3 doses of Duffelin. It can greatly improve the success rate of transplantation.

In this study, the incidence of neovascularization in infertility patients under hysteroscopy is $22.4 \%$, which is a significant feature of endometrial changes under hysteroscopy. Hysteroscopic neovascularization had moderate consistency with CE (Kappa value 0.46). Logsit regression analysis showed that the detection rate of neovascularization endometritis was up to 8 times higher than that of patients without neovascularization, which can be used as a characteristic index for physician to judge CE and improve the accuracy of CE diagnosis by hysteroscopy. Irregular hyperplasia and polypoid hyperplasia have little correlation with CE, but whether they affect embryo implantation and related mechanisms need to be further studied. In clinical work, endometrial polyps and endometrioid hyperplasia are often confused. The obvious difference is that endometrial polyps not only have different numbers of glands, stroma and blood vessels, but also need distinct pedicles and vascular axes. The microscopic appearance 
of endometrial polyp is that normal stroma and plasma cells can be seen around blood vessels.

In conclusion, the detection of endometrial congestion, microscopic polyps, or endometrial interstitial edema during hysteroscopy should be cautious of CE, but the overall accuracy of hysteroscopy in diagnosing CE is only $67 \%$, so it should not replace the choice of histological examination as a diagnostic tool. There was a moderate consistency between neovascularization and CE, and the detection rate of neovascularization endometritis was 8 times higher than that of patients without neovascularization, which could be used as a characteristic indicator for physician to judge chronic endometritis under microscope and improve the accuracy of CE diagnosis by hysteroscopy. Although uneven hyperplasia of uterus and polypoid hyperplasia have no statistical significance for the diagnosis of chronic endometrium, their influence on embryo implantation rate should be further studied.

\section{References}

[1] Chu, P., Arber, D. A. (2000). Paraffin-section detection of CD10 in 505 nonhematopoietic neoplasms. Frequent expression in renal cell carcinoma and endometrial stromal sarcoma [J]. American Journal of Clinical Pathology, 2000(3): 374.

[2] Ec Kert, L. O., Hawes, S. E., Wölner-Hanssen, P. K., et al. (2002). Endometritis: The clinical-pathologic syndrome [J]. American Journal of Obstetrics \& Gynecology, 2002, 186(4): 690-695.

[3] Chen, Y. Q., Fang, R. L., Luo, Y. N., et al. (2016). Analysis of the diagnostic value of CD138 for chronic endometritis, the risk factors for the pathogenesis of chronic endometritis and the effect of chronic endometritis on pregnancy: a cohort study [J]. BMC Women's Health, 2016, 16(1): 60.

[4] Bouet, P. E., et al. (2016). "Chronic endometritis in women with recurrent pregnancy loss and recurrent implantation failure: prevalence and role of office hysteroscopy and immunohistochemistry in diagnosis.” Fertility \& Sterility, (2016): 106-110.

[5] Song, D., Feng, X., Zhang, Q., et al. (2018). Prevalence and confounders of chronic endometritis in premenopausal women with abnormal bleeding or reproductive failure [J]. Reproductive Biomedicine Online, 2018:S147264831730456X.

[6] Darwish, A. M. (2016). Genital Infections and Infertility. Chronic Endometritis [J]. 2016, 10.5772/60460 (Chapter 3).

[7] Cicinelli, E., Vitagliano, A., Kumar, A., et al. (2019). Unified diagnostic criteria for chronic endometritis at fluid hysteroscopy: proposal and reliability evaluation through an international randomized-controlled observer study [J]. Fertility and Sterility, 2019, 112(1): 162-173.e2.

[8] Ettore, C., Leonardo, R., Roberto, N., et al. (2005). Endometrial micropolyps at fluid hysteroscopy suggest the existence of chronic endometritis [J]. Human Reproduction, 2005, 20(5): 1386-1389.

[9] Cicinelli, E., Ziegler, D. D., Nicoletti, R., et al. (2008). Chronic endometritis: correlation among hysteroscopic, histologic, and bacteriologic findings in a prospective trial with 2190 consecutive office hysteroscopies [J]. Fertility \& Sterility, 2008, 89(3): 677-684.

[10] Dsmm, A., Tcla, B., Yun, Z. C., et al. (2019). Correlation between hysteroscopy findings and chronic endometritis [J]. Fertility and Sterility, 2019, 111(4): 772-779.

[11] Jabbour, H. N., Sales, K. J., Catalano, R. D., et al. (2009). Inflammatory pathways in female reproductive health and disease [J]. Reproduction, 2009, 138(6): 903-19.

[12] Maybin, J. A., Critchley, H. O. D. (2015). Menstrual physiology: implications for endometrial pathology and beyond [J]. Human Reproduction Update, 2015(6): 748-761.

[13] Salamat, Shahriar M. (2010). Robbins and Cotran: Pathologic Basis of Disease [J]. Journal of Neuropathology \& Experimental Neurology, 2010, 69(2): 214-214.

[14] Bulmer, J. N., Lynn, M., Margaret, L., et al. (1991). Granulated lymphocytes in human endometrium: histochemical and immunohistochemical studies [J]. Human Reproduction, 1991(6): 791-798.

[15] Jones, R. K., Bulmer, J. N., Searle, R. F. (1998). Phenotypic and functional studies of leukocytes in human endometrium and endometriosis [J]. Human Reproduction Update, 1998(5): 702-709.

[16] Faas, M. M., Vos, P. D. (2017). Uterine NK cells and macrophages in pregnancy [J]. Placenta, 2017, 56.

[17] May, W., John, T., Farook, A. A. The distribution of endometrial leukocytes and their proliferation markers in trimegestone-treated postmenopausal women compared to the endometrium of the natural cycle: a dose-ranging study [J]. Human Reproduction (5): 1201-6.

[18] Armstrong, G. M., Maybin, J. A., Murray, A. A., et al. (2017). Endometrial apoptosis and neutrophil infiltration during menstruation exhibits spatial and temporal dynamics that are recapitulated in a mouse model [J]. Scientific Reports, 2017, 7(1): 17416 . 
[19] Sourima Biswas Shivhare, Judith N. Bulmer, Barbara A. Inne, Dharani K. Hapangama, Gendie E Lash, et al. (2015). Menstrual cycle distribution of uterine natural killer cells is altered in heavy menstrual bleeding [J]. Journal of Reproductive Immunology, 2015, 112: 88-94.

[20] Kotaro, K., Jinsuke, Y., Izumi, Y., et al. (2000). IL-15 Expression at Human Endometrium and Decidua [J]. Biology of Reproduction, 2000(3): 683-687.

[21] Kodama, T., Hara, T., Okamoto, E., et al. Characteristic changes of large granular lymphocytes that strongly express CD56 in endometrium during the menstrual cycle and early pregnancy [J]. Human Reproduction, (4): 1036.

[22] Takebayashi, A., Kimura, F., Kishi, Y., Ishida, M., Takahashi, A., Yamanaka, A., Takahashi, K., Suginami, H., Murakami, T. (2014). The association between endometriosis and chronic endometritis. PLoS One, 2014 Feb 18, 9(2): e88354. doi: 10.1371/journal.pone.0088354. PMID: 24558386; PMCID: PMC3928198. 